\title{
FLAT CIRCLE BUNDLES, PULLBACKS, AND THE CIRCLE MADE DISCRETE
}

\author{
JOHN OPREA AND DANIEL TANRÉ
}

Received 15 December 2004 and in revised form 5 June 2005

We use basic homotopical methods applied to Lie groups made discrete to prove that the real Euler class of a circle bundle vanishes if and only if the bundle is flat.

\section{Introduction}

The fact that flat principal circle bundles are characterized by having zero real Euler classes has proved important in recent years in understanding whether total spaces of vector bundles over nonnegatively curved manifolds must support metrics with nonnegative curvature as well (see Theorem 6.3 and the discussion afterwards). Unfortunately, the proofs of this fact of which we are aware use either sheaf-theoretic arguments that require a great deal of background somewhat out of the algebraic topological mainstream (see [4]) or the elaborate differential geometric machinery of Chern-Weil theory (see [7], e.g.). The purpose of this note is simply to observe that an enlightening topological proof exists that only relies on the notions of pullback and classifying map, as well the homological algebra surrounding these concepts. Moreover, as our proof demonstrates, the Euler class result is a direct manifestation of the circle's characterization as the only nontrivial compact connected Lie group with contractible universal cover. Finally, our proof provides a prime example of how homotopy theory and its associated algebra can have beautiful geometric consequences.

\section{Topological and homotopical background}

Good references for the following are $[3,9,10]$. A principal $G$-bundle $G \rightarrow P \stackrel{p}{\rightarrow} X$ is a fibre bundle that arises from a free smooth action of a Lie group $G$ on a smooth manifold $P$. That is, the base manifold $X$ is simply the quotient manifold $P / G$. We will always take $G$ to be a compact, connected Lie group. We are especially interested in the case where $G=S^{1}$, the circle group. For each $G$, there is a space $B G$, called the classifying space for $G$, which has the fundamental property that its loop space $\Omega B G$ is homotopy equivalent to $G$. The objects that $B G$ classifies, in fact, are the principal $G$-bundles. More specifically, 
3488 Flat circle bundles, pullbacks, and the circle made discrete

there is a bijection

$\{$ Isomorphism classes of principal $G$-bundles over $X\} \cong[X, B G]$,

where $[X, B G]$ denotes the set of homotopy classes of maps from $X$ to $B G$. If the bundle $G \rightarrow P \stackrel{p}{\rightarrow} X$ corresponds to a homotopy class represented by a map $\kappa: X \rightarrow B G$, then $\kappa$ is called a classifying map for the bundle. Note that a bundle is trivial (i.e., isomorphic to a product bundle $P \cong G \times X \stackrel{\mathrm{pr}_{X}}{\longrightarrow} X$ ) exactly when the classifying map is null-homotopic (i.e., homotopic to a constant map). A section of a principal bundle $G \rightarrow P \stackrel{p}{\rightarrow} X$ is a map $s: X \rightarrow P$ such that $p s=\mathrm{id}_{X}$. In Section 3, we will see that the existence of a section means that the principal bundle is trivial.

When $G=S^{1}$, something special happens. Namely, the classifying space $B S^{1}$ is an Eilenberg-MacLane space $K(\mathbb{Z}, 2)$, that is, $B S^{1}$ has second homotopy group $\pi_{2}\left(B S^{1}\right)=\mathbb{Z}$, and all other $\pi_{i}\left(B S^{1}\right)=0, i \neq 2$. In fact, $B S^{1}$ is known to be $\mathbb{C P}(\infty)$, the infinite complex projective space. For nice spaces (e.g., manifolds, CW-complexes), Eilenberg-MacLane spaces classify singular cohomology in the sense that

$$
H^{k}(X ; A) \cong[X, K(A, k)]
$$

where $H^{k}(X ; A)$ denotes the cohomology of $X$ with coefficients in the abelian group $A$, and $[X, K(A, k)]$ again denotes the set of homotopy classes of maps from $X$ to the Eilenberg-MacLane space $K(A, k)$, having $\pi_{k}(K(A, k))=A$ and $\pi_{i}(K(A, k))=0$ for $i \neq k$. (Also note that the loop space of an Eilenberg-MacLane space is an Eilenberg-MacLane space: $\Omega K(A, n)=K(A, n-1)$.) The bijection is given as follows. The universal coefficient theorem of algebraic topology shows that

$$
H^{k}(K(A, k) ; A)=\operatorname{Hom}\left(H_{k}(K(A, k) ; \mathbb{Z}), A\right)=\operatorname{Hom}(A, A) .
$$

The identity homomorphism id $: A \rightarrow A$ then corresponds to a characteristic element $\iota \in H^{k}(K(A, k) ; A)$. Now, given a map $f: X \rightarrow K(A, k)$, we can apply the induced homomorphism on cohomology to $\iota$ to get $f^{*}(\iota) \in H^{k}(X ; A)$. This is the bijection shown above.

By combining the discussions above, we see that when $G=S^{1}$, the isomorphism classes of principal circle bundles over a manifold $X$ are classified by $H^{2}(X ; \mathbb{Z})$. More specifically, if $\kappa: X \rightarrow B S^{1}$ classifies a principal $S^{1}$-bundle $S^{1} \rightarrow P \stackrel{p}{\rightarrow} X$, then the bundle is, in fact, classified by $\kappa^{*}(\iota) \in H^{2}(X ; \mathbb{Z})$. The Euler class of the bundle is then defined to be $e=\kappa^{*}(\iota) \in H^{2}(X ; \mathbb{Z})$. (The Euler class for principal circle bundles coincides with a more general definition for oriented vector bundles using the Thom class. This follows from the fact that circle bundles are the underlying principal bundles of oriented $\mathbb{R}^{2}$-vector bundles.) Therefore, the Euler class is the essential ingredient in the understanding of principal circle bundles. Noting that $S^{1} \cong U(1)$, we can also identify the Euler class of a principal $S^{1}$-bundle with the first Chern class of the associated complex line bundle-and this is an important reason why the Euler class holds interest for geometers.

An example of a principal bundle to keep in mind is the universal covering projection $\tilde{X} \rightarrow X$. This is a principal $\pi_{1}(X)$-bundle with classifying map $X \rightarrow B \pi_{1}(X)$. Now, when 
$G$ is a discrete group, we know that $B G=K(G, 1)$, so $B \pi_{1}(X)=K\left(\pi_{1}(X), 1\right)$ and the classifying map induces an isomorphism of fundamental groups (which can be obtained by attaching cells to $X$ to kill all higher homotopy groups).

The final bit of algebraic topological background needed is the universal coefficient theorem. There is a relation among cohomology groups, homology groups, and the Ext groups (of homological algebra). Namely, we have a split exact sequence (for an abelian group A)

$$
\operatorname{Ext}\left(H_{k-1}(X ; \mathbb{Z}), A\right) \longrightarrow H^{k}(X ; A) \longrightarrow \operatorname{Hom}\left(H_{k}(X ; \mathbb{Z}), A\right)
$$

An exact sequence of abelian groups (or, indeed, any central extension of an abelian group) $A \rightarrow B \rightarrow C$ has a fibration $K(A, 1) \rightarrow K(B, 1) \rightarrow K(C, 1)$ associated with it which is a homotopy-analog to a principal bundle. (Indeed, the long exact homotopy sequence described in Properties 3.1(3) is the original short exact sequence.) Just as for principal bundles, the fibration has a classifying map $K(C, 1) \rightarrow K(A, 2)$ corresponding to an element of $H^{2}(K(C, 1) ; A)=H^{2}(C ; A)$. The universal coefficient theorem then gives

$$
\operatorname{Ext}(C, A)=\operatorname{Ext}\left(H_{1}(K(C, 1) ; \mathbb{Z}) ; A\right)=H^{2}(C ; A),
$$

since $H_{2}(C ; \mathbb{Z})=0$ for a finitely generated abelian group $C$ (see [2], e.g.). This is the usual extension group which classifies abelian extensions of $C$ by $A$. Having this foundational material, we can now consider one of the main subjects of the paper.

\section{Pullbacks}

There are two types of pullbacks that will be important for us. The topological pullback, or simply pullback is defined as follows: given two maps $f: X \rightarrow B$ and $g: Y \rightarrow B$, we form the space

$$
\mathbf{P}=\{(x, y) \in X \times Y \mid f(x)=g(y)\}
$$

and call it the pullback of $f$ and $g$. The key property of a pullback is that, given $h: Z \rightarrow X$ and $k: Z \rightarrow Y$ with $f h=g k$, then there exists a (unique) map $\omega: Z \rightarrow \mathbf{P}$ making the following diagram commutative, where $\bar{f}$ and $\bar{g}$ are the canonical projections:

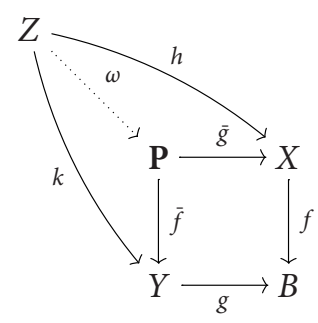

The most important homotopical construction for our purposes is the homotopy pullback. Given two maps $f: X \rightarrow B$ and $g: Y \rightarrow B$, we form the space

$$
\mathscr{P}=\left\{(x, \gamma, y) \in X \times B^{I} \times Y \mid f(x)=\gamma(0), g(y)=\gamma(1)\right\}
$$


and call it the homotopy pullback of $f$ and $g$. Many results about homotopy pullbacks may be found in [5], but we will require only the following.

Properties 3.1. The following properties hold.

(1) Given $h: Z \rightarrow X$ and $k: Z \rightarrow Y$ with $f h \simeq g k$, then there exists a map $\omega: Z \rightarrow \mathscr{P}$ making the following diagram homotopy commutative, where $\bar{f}$ and $\bar{g}$ are the canonical projections:

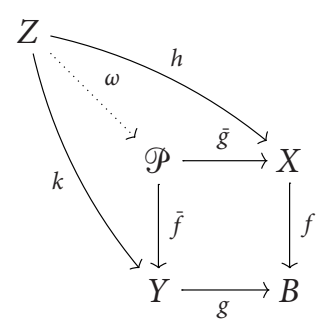

(2) Replacing any of the spaces or maps by homotopy equivalent ones in the initial diagram produces the same homotopy pullback up to homotopy.

(3) If $X$, say, is contractible, then $\mathscr{P}$ is the homotopy fibre of the map $g: Y \rightarrow B$. For instance, if we take $X=P B=\left\{\gamma: I \rightarrow B \mid \gamma(0)=b_{0}\right\}$, the contractible path space over $B$ consisting of paths in $B$ starting at a fixed basepoint, and $f(\gamma)=\gamma(1)$, then we obtain a fibration up to homotopy $\mathscr{P} \rightarrow Y \stackrel{g}{\rightarrow} B$. (A fibration is a map which satisfies the homotopy lifting property (see [9] or [10]), while a fibration up to homotopy means that spaces and maps may be replaced by homotopically equivalent ones which give an actual fibration.) We then call $\mathscr{P}=F_{g}$ the homotopy fibre of $g$. The homotopy lifting property may be interpreted as the following property. If $F \stackrel{j}{\rightarrow} E \stackrel{p}{\rightarrow} B$ is a fibration, then given any map $f$ : $X \rightarrow E$ such that $p \circ f$ is null-homotopic, there exists a "lifting" $g: X \rightarrow F$ with $j \circ g \simeq f$. (Here, $\simeq$ denotes "homotopic to.")

(4) By continuing this process of taking homotopy fibres, we obtain a sequence of spaces (called the Puppe sequence):

$$
\cdots \longrightarrow \Omega F_{g} \longrightarrow \Omega Y \stackrel{\Omega g}{\longrightarrow} \Omega B \longrightarrow F_{g} \longrightarrow Y \longrightarrow g B
$$

where any three consecutive spaces form a fibration up to homotopy (with any two consecutive maps being null-homotopic). The crucial property of the Puppe sequence is that it is exact in the sense that, for any space $Y$, the following sequence of homotopy sets is exact:

$$
\cdots \longrightarrow\left[X, \Omega F_{g}\right] \longrightarrow[X, \Omega Y] \stackrel{\Omega g_{\#}}{\longrightarrow}[X, \Omega B] \longrightarrow\left[X, F_{g}\right] \longrightarrow[X, Y] \stackrel{g_{\#}}{\longrightarrow}[X, B]
$$


In particular, if $X=S^{0}$ (the 0 -sphere), then we obtain the long exact sequence of homotopy groups associated to a fibration (see [9] or [10]):

$$
\cdots \longrightarrow \pi_{k+1}(B) \longrightarrow \pi_{k}\left(F_{g}\right) \longrightarrow \pi_{k}(Y) \longrightarrow \pi_{k}(B) \longrightarrow \pi_{k-1}\left(F_{g}\right) \longrightarrow \cdots \longrightarrow \pi_{0}(B) .
$$

The homomorphisms $\pi_{j+1}(B) \rightarrow \pi_{j}\left(F_{g}\right)$ are called connecting homomorphisms.

The map preceding a sequence of three spaces (as we go from right to left) in the Puppe sequence is said to classify the sequence because the sequence arises as a homotopy pullback of the map. So the homotopy class of the classifying map determines the homotopy type of the homotopy pullback. For instance, $g$ classifies $\Omega B \rightarrow F_{g} \rightarrow Y$.

(5) If $f: X \rightarrow B$ is a fibration, then $\mathscr{P}$ has the homotopy type of the ordinary topological pullback P. Because $f: P B \rightarrow B$ as above is a fibration for any $B$, the homotopy pullback of all classifying maps in our sense is fibrations.

Example 3.2. Suppose $G \rightarrow P \stackrel{P}{\rightarrow} X$ is a principal bundle with classifying map $\kappa: X \rightarrow B G$. Since $\Omega B G \simeq G$, the Puppe sequence extends to $\cdots \rightarrow G \rightarrow P \rightarrow X \rightarrow B G$ with accompanying exact sequence

$$
\cdots \longrightarrow[X, P] \stackrel{p_{\#}}{\longrightarrow}[X, X] \stackrel{\kappa_{\#}}{\longrightarrow}[X, B G] .
$$

Suppose the bundle has a section $s: X \rightarrow P$. Then there is an induced map $s_{\#}:[X, X] \rightarrow$ $[X, P]$ with $p_{\#} \circ s_{\#}=\operatorname{id}_{[X, X]}$. Hence,

$$
\kappa=\kappa_{\#}\left(\operatorname{id}_{X}\right)=\kappa_{\#}\left(p_{\#} \circ s_{\#}\left(\operatorname{id}_{X}\right)\right)=\left(\left(\kappa_{\#} p_{\#}\right) \circ s_{\#}\right)\left(\operatorname{id}_{X}\right)=*,
$$

since $\kappa \circ p$ is null-homotopic. Since the classifying map is null-homotopic, the principal bundle is trivial. Thus, principal bundles with sections must be trivial bundles.

\section{Flat bundles and Lie groups made discrete}

A principal $G$-bundle $G \rightarrow P \rightarrow X$ (with classifying map $\kappa: X \rightarrow B G$ ) is called flat if the classifying map factors up to homotopy as

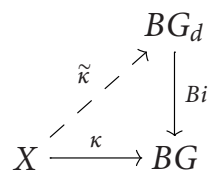

where $G_{d}$ is the group $G$ (but with the discrete topology), $i: G_{d} \rightarrow G$ is the identity map, and $B G_{d}$ is the classifying space of $G_{d}$. Such a factorization has the effect of reducing the structure group of the bundle to a discrete group, so that any associated vector bundle has a connection with curvature zero. This is what is meant by "flat." Also, it should 
be mentioned that there is an interesting conjecture concerning the relationship in homology between $B G_{d}$ and $B G$; namely, that $B i_{*}: H_{*}\left(B G_{d} ; A\right) \rightarrow H_{*}(B G ; A)$ should be an isomorphism for all finite $A$. See [6] for a proof when $G$ is solvable.

Note that since $G_{d}$ is a discrete group, the classifying space $B G_{d}=K\left(G_{d}, 1\right)$ is the Eilenberg-MacLane space having its single nontrivial homotopy group $G_{d}$ in degree one. The following result is the first step in reducing the understanding of $G_{d}$ to that of its universal covering group made discrete.

Proposition 4.1. Let $G$ be a connected Lie group, and let $\pi: U \rightarrow G$ denote the universal covering. Then, $U_{d}$ is a pullback of $\pi$ along the map $i: G_{d} \rightarrow G$.

Proof. Note first that making $U$ discrete unravels all of the topology linking the fibres of the universal covering of $G$. Hence, as a (discrete) space, $U_{d}=G_{d} \times \pi_{1}(G)$. Now, discrete spaces are characterized by their cardinalities, and the pullback $\mathbf{P}$ of $\pi$ along the map $i$ : $G_{d} \rightarrow G$ clearly has the right cardinality. Therefore, the proof of the result simply consists of showing that $\mathbf{P}$ has the discrete topology.

Now, because $\pi: U \rightarrow G$ is a principal $\pi_{1}(G)$-bundle, its pullback along $i$ is one also. Because $\pi: U \rightarrow G$ is surjective, there is a set map $s: G \rightarrow U$ with $\pi \circ s=\mathrm{id}_{G}$. But, $G_{d}$ has the discrete topology, so $s$, considered as a map $s: G_{d} \rightarrow U$, is continuous. By Example 3.2, the principal bundle is trivial, so we obtain $\mathbf{P}=G_{d} \times \pi_{1}(G)$. Hence, $\mathbf{P}$ has the discrete topology and $\mathbf{P}=U_{d}$.

Remark 4.2. We can also prove the proposition by letting $q: \mathbf{P} \rightarrow G_{d}$ and $r: \mathbf{P} \rightarrow U$ be the continuous projections and considering the inverse images $q^{-1}(g)=\{(g, x) \mid \pi(x)=$ $g$ \} and $r^{-1}(A)$, where $A$ is an open set in $U$ about $x$ containing no other point in the fibre above $g$. These sets are open by continuity and the fact that $g \in G_{d}$ is open. Hence, $q^{-1}(g) \cap r^{-1}(A)=\{(g, x)\}$ is open too and, thus, $\mathbf{P}$ has the discrete topology. Therefore, $\mathbf{P}=G_{d} \times \pi_{1}(G)=U_{d}$.

\section{The case of the circle group}

Let $G=S^{1}$ with universal cover $\mathbb{R}$, and note that $B S^{1}=K(\mathbb{Z}, 2), B S_{d}^{1}=K\left(S_{d}^{1}, 1\right)$, and $B \mathbb{R}_{d}=K\left(\mathbb{R}_{d}, 1\right)$. Now, consider the following pullback diagram arising as in Proposition 4.1:

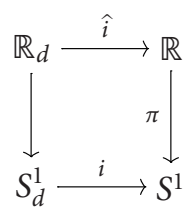

Of course, the homomorphisms of groups shown are the usual ones making the sequence $\mathbb{Z} \rightarrow \mathbb{R} \rightarrow S^{1}$ exact. Note that the pullback property guarantees that the fibre of $\mathbb{R}_{d} \rightarrow S_{d}^{1}$ is $\mathbb{Z}$ included in $\mathbb{R}_{d}$ in the standard way and that the restriction of $\hat{i}$ to fibres is the identity. But now we notice an amazing fact. Because $\mathbb{R} \rightarrow S^{1}$ is a fibration with $\mathbb{R}$ contractible, by Properties 3.1(3), $\mathbb{R}_{d}$ is the homotopy fibre of $S_{d}^{1} \rightarrow S^{1}$. Therefore, $i: S_{d}^{1} \rightarrow S^{1}$ is the classifying map for $\mathbb{Z} \rightarrow \mathbb{R}_{d} \rightarrow S_{d}^{1}$, and the same holds for $B S_{d}^{1} \rightarrow B S^{1}$ in the sequence of 
classifying spaces:

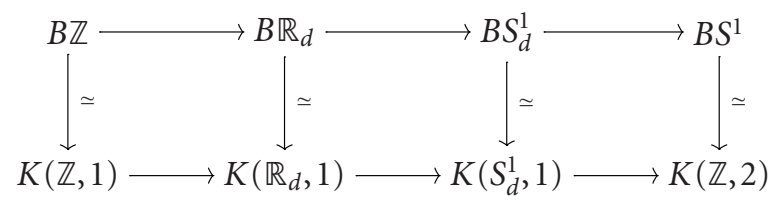

Remark 5.1. Note that the first two maps are determined by the corresponding homomorphisms of groups. The last map also comes from the sequence of discrete groups; it is the class in $\operatorname{Ext}\left(S_{d}^{1}, \mathbb{Z}\right) \subseteq H^{2}\left(S_{d}^{1} ; \mathbb{Z}\right)$ classifying the central extension $\mathbb{Z} \rightarrow \mathbb{R}_{d} \rightarrow S_{d}^{1}$ (see [2]).

Proposition 5.2. There exists a map $\phi: K(\mathbb{Z}, 2) \rightarrow K\left(\mathbb{R}_{d}, 2\right)$, so that the sequence of fibrations (5.2) may be extended to a sequence of fibrations

$$
K(\mathbb{Z}, 1) \longrightarrow K\left(\mathbb{R}_{d}, 1\right) \longrightarrow K\left(S_{d}^{1}, 1\right) \longrightarrow K(\mathbb{Z}, 2) \stackrel{\phi}{\longrightarrow} K\left(\mathbb{R}_{d}, 2\right)
$$

Proof. This is, in fact, a standard result in topology (see [1, Theorem 7.1]), but we give a proof to illustrate the beautiful connections between topology and algebra outlined in Section 2.

Let $\phi$ correspond to the usual inclusion of the integers into the reals, $\mathbb{Z} \rightarrow \mathbb{R}_{d}$. Of course, this is the induced homomorphism of the map at the beginning of the sequence $K(\mathbb{Z}, 1) \rightarrow K\left(\mathbb{R}_{d}, 1\right)$ as well. The homomorphism $\phi$ also gives a map $K(\mathbb{Z}, 2) \rightarrow K\left(\mathbb{R}_{d}, 2\right)$ which is $\phi$ on homotopy groups.

To see that we get a fibration on the right end, let $F$ denote the homotopy fibre of $\phi$. We must show that $F \rightarrow K(\mathbb{Z}, 2)$ is precisely the map $K\left(S_{d}^{1}, 1\right) \rightarrow K(\mathbb{Z}, 2)$ in the original fibration sequence corresponding to the group extension $\mathbb{Z} \rightarrow \mathbb{R}_{d} \rightarrow S_{d}^{1}$ as discussed in Remark 5.1.

The long exact sequence in homotopy associated to the fibration $F \rightarrow K(\mathbb{Z}, 2) \rightarrow K\left(\mathbb{R}_{d}, 2\right)$ shows immediately that $F \simeq K\left(S_{d}^{1}, 1\right)$ since (because $\mathbb{Z} \rightarrow \mathbb{R}_{d}$ is injective) the only nontrivial homotopy group appears in degree one and is a quotient of the standard inclusion $\mathbb{Z} \rightarrow \mathbb{R}_{d}$ (which defines $\phi$ ). We now extend to a Puppe sequence (see Properties 3.1(4))

$$
K(\mathbb{Z}, 1) \stackrel{\Omega \phi}{\longrightarrow} K\left(\mathbb{R}_{d}, 1\right) \longrightarrow F=K\left(S_{d}^{1}, 1\right) \longrightarrow K(\mathbb{Z}, 2) \stackrel{\phi}{\longrightarrow} K\left(\mathbb{R}_{d}, 2\right),
$$

where we can see that the homomorphisms corresponding to the first two maps are the usual ones. The first is just a loop of $\phi$, so that is clear. The second map is just a realization of the connecting homomorphism $\pi_{2}\left(K\left(\mathbb{R}_{d}, 2\right)\right) \rightarrow \pi_{1}\left(K\left(S_{d}^{1}, 1\right)\right)=F$ in the homotopy sequence by spaces $K\left(\mathbb{R}_{d}, 1\right)=\Omega K\left(\mathbb{R}_{d}, 2\right) \rightarrow K\left(S_{d}^{1}, 1\right)$. Since the connecting 
homomorphism was the usual group projection $\mathbb{R}_{d} \rightarrow S_{d}^{1}$ onto the quotient $S_{d}^{1}=\mathbb{R}_{d} / \mathbb{Z}$, the second map is the usual one too. The map $F \rightarrow K(\mathbb{Z}, 2)$ is a classifying map for the fibration $K(\mathbb{Z}, 1) \rightarrow K\left(\mathbb{R}_{d}, 1\right) \rightarrow F=K\left(S_{d}^{1}, 1\right)$, since the Puppe sequence is constructed by taking consecutive homotopy fibres. But the fibration is determined by the group extension $\mathbb{Z} \rightarrow \mathbb{R}_{d} \rightarrow S_{d}^{1}$, since the spaces are Eilenberg-MacLane spaces in degree one. Indeed, the classifying map corresponds to an element in $\operatorname{Ext}\left(S_{d}^{1}, \mathbb{Z}\right) \subseteq H^{2}\left(S_{d}^{1} ; \mathbb{Z}\right)$ classifying the group extension. But the group extension is the usual one, so the element, and hence the classifying map $F \rightarrow K(\mathbb{Z}, 2)$ are the usual ones as well.

\section{Flat $S^{1}$-bundles and the Euler class}

A principal $S^{1}$-bundle $\xi: S^{1} \rightarrow P \rightarrow X$ is classified by a map $\kappa: X \rightarrow B S^{1}=K(\mathbb{Z}, 2)$. As discussed in Section 2, this map corresponds to a degree-2 cohomology class by the standard identification $[X, K(\mathbb{Z}, 2)] \cong H^{2}(X ; \mathbb{Z})$. Indeed, there is an element $\iota \in H^{2}(K(\mathbb{Z}, 2) ; \mathbb{Z})$ corresponding to the identity homomorphism id: $\mathbb{Z} \rightarrow \mathbb{Z}$, and the Euler class $e(\xi) \in H^{2}(X ; \mathbb{Z})$ has $e(\xi)=\kappa^{*}(\iota)$. As we have said earlier, $e(\xi)$ characterizes the homotopy class of $\kappa$, so it serves to classify principal $S^{1}$-bundles over $X$. A real Euler class $e(\xi)_{\mathbb{R}_{d}}$ is obtained by simply extending coefficients to $H^{2}\left(X ; \mathbb{R}_{d}\right)$. This is equivalent to composing with the map $\phi: K(\mathbb{Z}, 2) \rightarrow K\left(\mathbb{R}_{d}, 2\right)$ corresponding to the usual inclusion $\mathbb{Z} \rightarrow \mathbb{R}_{d}$. Again, we have $\left[X, K\left(\mathbb{R}_{d}, 2\right)\right] \cong H^{2}\left(X ; \mathbb{R}_{d}\right)$ obtained by pulling back a class $\hat{\imath}$ corresponding to id: $\mathbb{R}_{d} \rightarrow$ $\mathbb{R}_{d}$. (Note that pulling $\hat{\imath}$ back to $K(\mathbb{Z}, 2)$ gives the class in $H^{2}\left(K(\mathbb{Z}, 2) ; \mathbb{R}_{d}\right)$ corresponding to the usual inclusion $\mathbb{Z} \rightarrow \mathbb{R}_{d}$.) We can now prove the main result using only the standard fibration and pullback theory presented above.

Theorem 6.1. The real Euler class $e(\xi)$ is zero if and only if $\xi$ is flat.

Proof. With the notation above, consider the following fibration diagram, which we will use for both parts of the proof (here we write $B S^{1}$ for $K(\mathbb{Z}, 2)$ and $B S_{d}^{1}$ for $K\left(S_{d}^{1}, 1\right)$ to relate back to the definition of flat bundle):

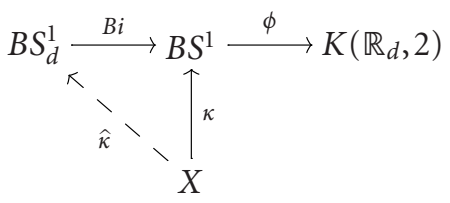

Suppose $\xi$ is flat. Then $\widehat{\kappa}$ exists by definition and we have $\phi \kappa \simeq \phi B i \widehat{\kappa} \simeq *$ since $\phi B i \simeq *$. But $\phi \kappa$ represents the real Euler class, so we obtain $e(\xi)_{\mathbb{R}_{d}}=0$.

On the other hand, suppose $e(\xi)_{\mathbb{R}_{d}}=0$. Then $\phi \kappa \simeq *$. But then Properties 3.1(3) allows us to factor $\kappa$ through the fibre as shown in the diagram: $B i \tilde{\kappa} \simeq \kappa$. By definition, this means that the bundle is flat.

Of course, when $X$ has finite type, for an integral cohomology class $e$, we can identify the conditions that $e$ vanishes when coefficients are extended to the reals and that $e$ is torsion. Therefore, we have the following corollary.

CoROLlaRY 6.2. If X has finite type, then the Euler class $e(\xi)$ is torsion if and only if $\xi$ is flat. 
We end by noting an interesting result that uses Corollary 6.2. In an attempt to create new examples of nonnegatively curved manifolds, geometers have considered the question of whether total spaces of vector bundles over nonnegatively curved manifolds also carry metrics of nonnegative sectional curvature. In [8], Özaydin and Walschap gave examples showing this is not always the case and a fundamental ingredient in their proof is the following. (Recall that a compact manifold is flat if its sectional curvature is constantly zero. Also recall that an oriented $\mathbb{R}^{2}$-vector bundle has a circle bundle as underlying principal bundle and the Euler class comes from that circle bundle.)

Theorem 6.3 (see [8]). Let $\xi: \mathbb{R}^{2} \rightarrow E \rightarrow M$ be an oriented vector bundle, where $M^{n}$ is a compact flat manifold. Then, $E$ admits a complete nonnegatively curved metric if and only if the rational Euler class $e(\xi)_{\mathbb{Q}}$ is zero.

Because oriented $\mathbb{R}^{2}$-bundles over $T^{2}$ are classified (in terms of their associated principal circle bundles) by Euler classes $e \in\left[T^{2}, B S^{1}\right] \cong H^{2}\left(T^{2} ; \mathbb{Z}\right)=\mathbb{Z}$, by Theorem 6.3 , only the zero Euler class gives a total space with nonnegative curvature. But this is simply $T^{2} \times \mathbb{R}^{2}$, the total space of the trivial bundle. All other total spaces cannot carry metrics of nonnegative curvature. These are the examples of Özaydin and Walschap.

\section{References}

[1] P. J. Hilton, Homotopy Theory and Duality, Gordon and Breach Science, New York, 1965.

[2] P. J. Hilton and U. Stammbach, A Course in Homological Algebra, 2nd ed., Graduate Texts in Mathematics, vol. 4, Springer, New York, 1997.

[3] D. Husemoller, Fibre Bundles, 3rd ed., Graduate Texts in Mathematics, vol. 20, Springer, New York, 1994.

[4] F. Kamber and Ph. Tondeur, Flat bundles and characteristic classes of group-representations, Amer. J. Math. 89 (1967), no. 4, 857-886.

[5] M. Mather, Pull-backs in homotopy theory, Canad. J. Math. 28 (1976), no. 2, 225-263.

[6] J. Milnor, On the homology of Lie groups made discrete, Comment. Math. Helv. 58 (1983), no. 1, $72-85$.

[7] S. Morita, Geometry of Characteristic Classes, Translated from the 1999 Japanese original. Translations of Mathematical Monographs, vol. 199, Iwanami Series in Modern Mathematics, American Mathematical Society, Rhode Island, 2001.

[8] M. Özaydin and G. Walschap, Vector bundles with no soul, Proc. Amer. Math. Soc. 120 (1994), no. 2, 565-567.

[9] E. H. Spanier, Algebraic Topology, Corrected reprint, Springer, New York, 1981.

[10] G. W. Whitehead, Elements of Homotopy Theory, Graduate Texts in Mathematics, vol. 61, Springer, New York, 1978.

John Oprea: Department of Mathematics, Cleveland State University, Cleveland, OH 44115-2214, USA

E-mail address: oprea@math.csuohio.edu

Daniel Tanré: Département de Mathématiques, Université de Lille 1, 59655 Villeneuve d’Ascq Cedex, France

E-mail address: daniel.tanre@univ-lille1.fr 


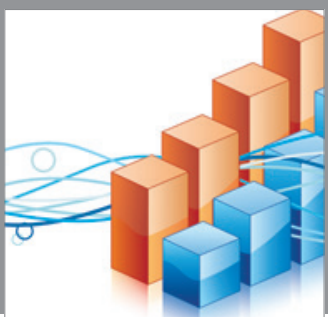

Advances in

Operations Research

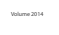

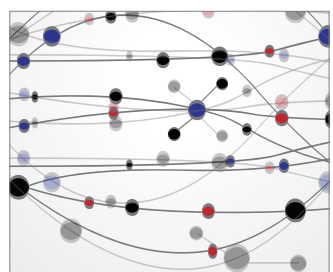

\section{The Scientific} World Journal
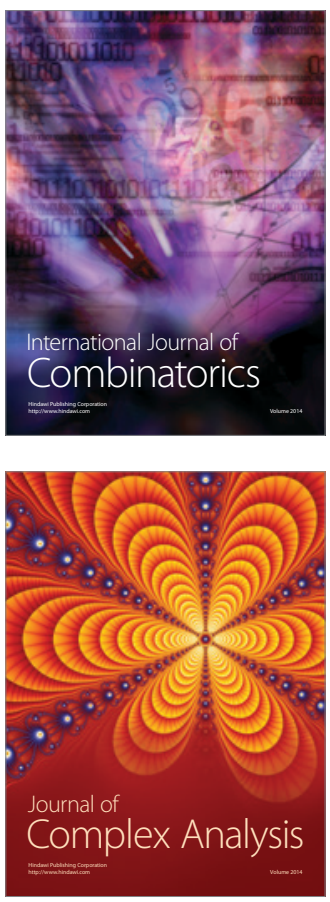

International Journal of

Mathematics and

Mathematical

Sciences
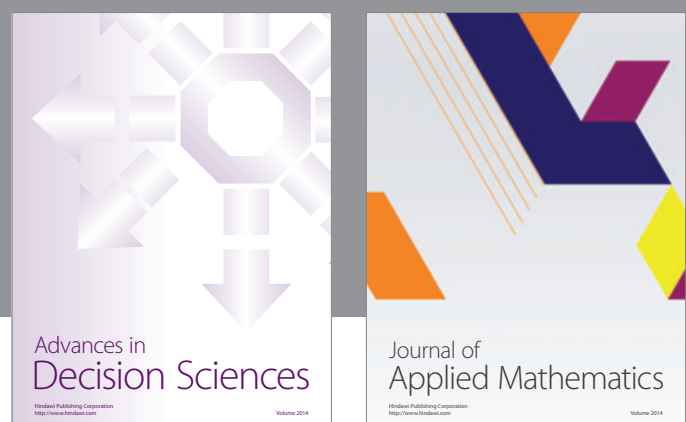

Journal of

Applied Mathematics
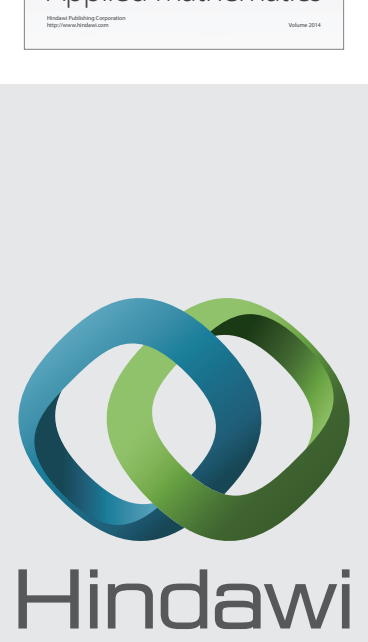

Submit your manuscripts at http://www.hindawi.com
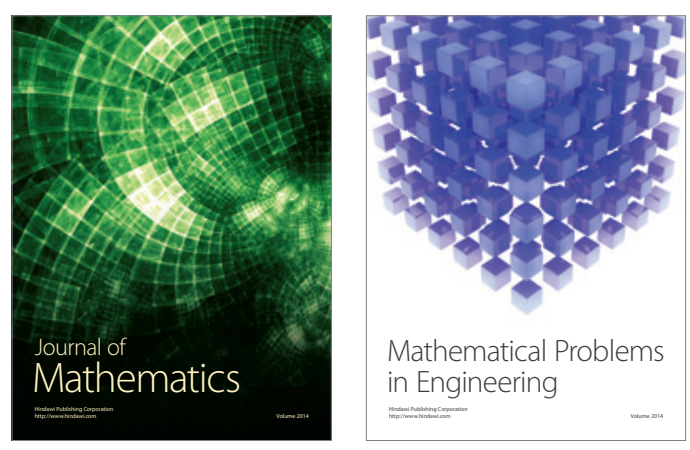

Mathematical Problems in Engineering
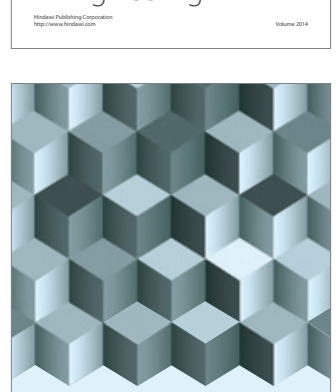

Journal of

Function Spaces
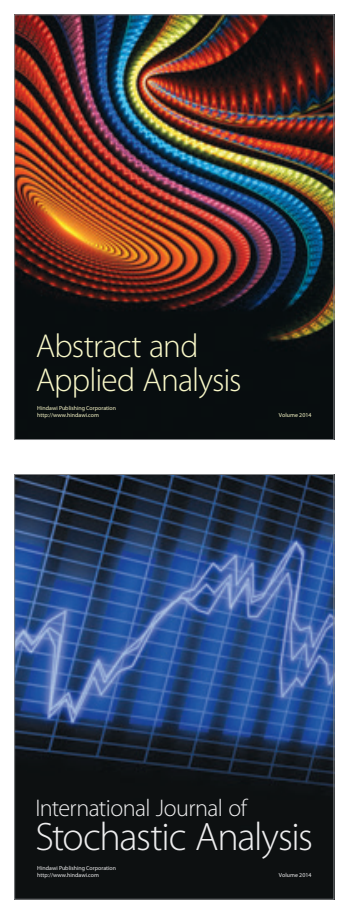

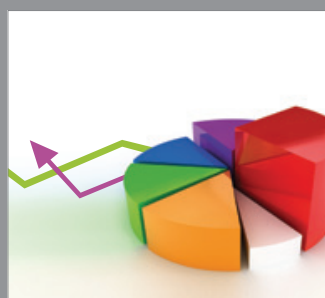

ournal of

Probability and Statistics

Promensencen
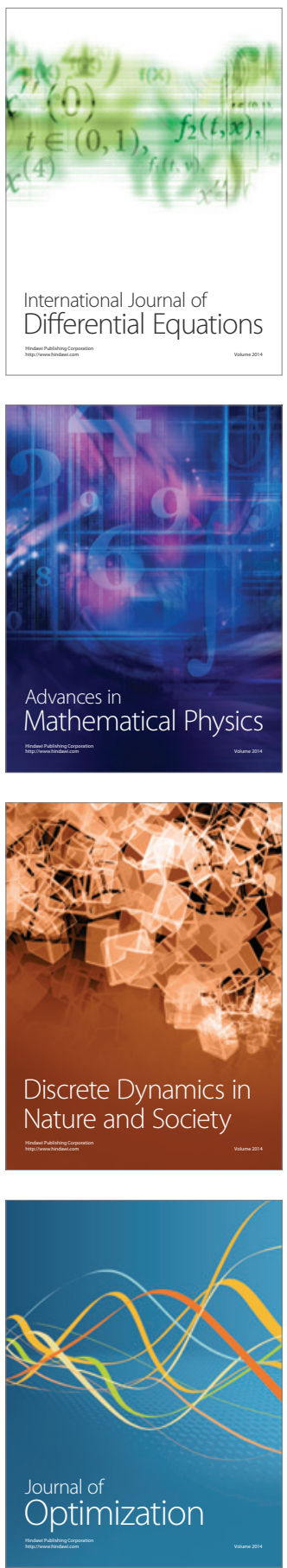